\title{
ON MAXIMAL NONPARABOLIC NORMAL SUBGROUPS OF THE MODULAR GROUP
}

\author{
A. W. MASON AND N. K. DICKSON
}

\begin{abstract}
Let $F$ be a free normal subgrcup of finite index in the modular group $\Gamma$ with $F \neq \Gamma^{\prime} . F^{\prime}$ is known to be a nonparabolic normal subgroup of $\Gamma$. We show that, with two exceptions, there exists another nonparabolic normal subgroup of $\Gamma$ properly containing $F^{\prime}$.
\end{abstract}

1. Introduction. A subgroup of the modular group $\Gamma$ is said to be parabolic if it contains parabolic elements, i.e. conjugates of nonzero powers of $u$, where $u= \pm\left[\begin{array}{ll}1 & 1 \\ 0 & 1\end{array}\right]$. A maximal nonparabolic subgroup of $\Gamma$ ([4], [11]) is a subgroup which contains no parabolic elements and which is maximal with respect to this property. In this note we are concerned with those nonparabolic normal subgroups $M$ of $\Gamma$ which are maximal in the sense that any normal subgroup of $\Gamma$ properly containing $M$ is parabolic. We call such subgroups $m$-subgroups.

Throughout $F$ denotes a free normal subgroup of finite index in $\Gamma$. (There are only two proper normal subgroups of $\Gamma$ which are not free [9, p. 143].) It is known [5] that $F^{\prime}$ is a nonparabolic normal subgroup of $\Gamma$, provided $F \neq \Gamma^{\prime}$. It has also been shown ([4], [11]) that $F^{\prime}$ is an $m$-subgroup when $F=\Gamma(2)$ or $\Gamma(3)$, where $\Gamma(n)$ is the principal congruence subgroup of level $n$. ( $n$ is a positive integer.) In this note we show that, if $F \neq \Gamma(2)$ or $\Gamma(3)$, then $F^{\prime}$ is not an $m$-subgroup. By virtue of ([6], [7]) the proof of this result (\$2) depends upon the existence $(\$ 3)$ of particular integral representations of certain Frobenius groups. (Note that it has already been shown in [11] using the results of [6] that $\Gamma(5)^{\prime}$ is not an $m$-subgroup.)

It is clear that each $F^{\prime}$ is contained in some $m$-subgroup, provided $F \neq \Gamma^{\prime}$. Whether or not every $m$-subgroup is of this type remains an open question. In this connection Schupp [10] establishes the existence of uncountably many infinite simple groups each of which is generated by a pair of elements one of order two and the other of order three. Unfortunately, it is not yet known whether the order of the product of the pair of elements is finite or infinite.

As usual we denote the sets of integers, rational numbers and complex numbers by $\mathbf{Z}, \mathbf{Q}$ and $\mathbf{C}$, respectively.

Received by the editors June 21, 1978.

AMS (MOS) subject classifications (1970). Primary 20H05, 20H10.

Key words and phrases. Modular group, nonparabolic, maximal, just-infinite. 
2. The principal results. It is well known that $F$ has a presentation of the type

$$
F=\left\langle p_{1}, \ldots, p_{t}, a_{1}, b_{1}, \ldots, a_{g}, b_{g}: p_{1} \ldots p_{t} \prod_{i=1}^{g}\left[a_{i}, b_{i}\right]=1\right\rangle,
$$

where $p_{1}, \ldots, p_{t}$ are parabolic. Every parabolic element of $F$ is conjugate in $F$ to a power of one (and only one) of the $p_{i} \cdot t(=t(F))$ is the parabolic class number (or cusp number) and $g$ the genus of $F$. The rank of $F$ by [6] or [9, p. $144]$ is $1+\mu / 6$, where $\mu=(\Gamma: F)(=\mu(F))$.

The level of $F$ is the least positive integer $n$ for which $\Delta(n) \leqslant F$, where $\Delta(n)$ is the normal closure in $\Gamma$ of $u^{n} . \Delta(n)$ is the normal closure in $F$ of the set $\left\{p_{1}, \ldots, p_{t}\right\}$ by $[6]$.

If $g \in \Gamma$ and $1 \leqslant i \leqslant t$, then

$$
g p_{i} g^{-1}=f p_{j} f^{-1},
$$

where $f \in F$ and $j$ is uniquely determined by $g$ and $i$. In this way [6, p. 454] we may regard $\Gamma / F^{*}$ as a transitive permutation group on the "symbols" $p_{1}, \ldots, p_{t}$, which is isomorphic (as a permutation group) to the permutation representation of $\Gamma / F^{*}$ on the left cosets of $F^{u} / F^{*}$, where $F^{*}$ is the largest normal subgroup of $\Gamma$ contained in $F^{u}=\langle F, u\rangle$. It is known (see, for example, [7]) that $t\left(F^{*}\right)=t(F)$ from which it follows that, if $F \neq \Gamma^{\prime}$, then $F^{*}$ is free and $F^{*} \neq \Gamma^{\prime}$.

$\Gamma$ acts by conjugation on $P=F^{\prime} \cdot \Delta(n) / F^{\prime}$, which is generated by $p_{1}, \ldots, p_{t}\left(\bmod F^{\prime}\right)$, and under this action (which "extends" in a natural way the action of $\Gamma / F^{*}$ on $p_{1}, \ldots, p_{t}$ described above) $[6$, p. 454] $P$ becomes a faithful $\mathbf{Z} G$-module of rank $t-1$, where $G=\Gamma / F^{*}$.

LEMMA 2.1. Let $E$ be a normal subgroup of $\Gamma$ properly containing $F$. Then $F^{\prime} \neq E^{\prime} \cap F$.

Proof. We suppose that $F^{\prime}=E^{\prime} \cap F$. If $E$ is not free, $\left(\Gamma: E^{\prime}\right)$ is finite [9, p. 143]. Hence $F / F^{\prime}\left(\cong F \cdot E^{\prime} / E^{\prime}\right)$ is a subgroup of the free abelian group $E / E^{\prime}$. By considering ranks we deduce that $1+\mu(F) / 6 \leqslant 1+\mu(E) / 6$, which implies that $F=E$.

Let $S$ be the set of all positive integers $q$, where $q=p, p$ prime, $p \equiv 1(\bmod$ 6) or $q=p^{2}, p$ prime, $p \equiv-1(\bmod 6)$.

LEMMA 2.2. Assume $F^{*} \neq \Gamma(2), \Gamma(3)$. Then $F^{u}$ is a maximal subgroup of $\Gamma$ if and only if $F^{*}$ has level 6 and cusp number $q$, for some $q \in S$.

Proof. Assume that $F^{u}$ is maximal. Then $F \neq \Gamma^{\prime}$, since $\left(\Gamma^{\prime}\right)^{u}=\Gamma$, and so $F^{*}$ is free. It follows from [7, Theorem 4.2] that $n_{1} \mid 6$ and $t\left(F^{*}\right)(=t(F))=p^{s}$, where $n_{1}$ is the level of $F^{*}, p$ is a prime and $s=\operatorname{ord}_{n_{1}}(p)$. If $n_{1}=2, F^{*}=\Gamma(2)$ and if $n_{1}=3, F^{*}=\Gamma(3)$. Hence $n_{1}=6$ and $t\left(F^{*}\right) \in S$.

Conversely, if $F^{*}$ has level 6 and cusp number $q$, it can be shown [8] that 
$F^{u}=\left(F^{*}\right)^{u}$ is maximal. (For any $q \in S$ there exists at least one normal subgroup of level 6 and cusp number $q[8]$.)

It is easily verified that $F^{u}$ is maximal when $F^{*}=\Gamma(2)$ or $\Gamma(3)$. There are only five normal subgroups $F$ for which $F^{*}=\Gamma(2)$ or $\Gamma(3)$ by [6].

THEOREM A. Let $F$ be a free normal subgroup of finite index in $\Gamma$, with $F \neq \Gamma(2)$ or $\Gamma(3)$. Then $F^{\prime}$ is not an $m$-subgroup.

Proof. We may assume that $F \neq \Gamma^{\prime}$ since $\Gamma^{\prime \prime}=\Delta(6)$ by [8]. $F^{\prime}$ is then a nonparabolic normal subgroup of $\Gamma$ by [5]. Let $F_{0}$ be any normal subgroup of $\Gamma$ which contains $F^{\prime}$ and let $P_{0}=F^{\prime} \cdot\left(\Delta(n) \cap F_{0}\right) / F^{\prime}$. It is clear that $F_{0}$ is parabolic if and only if $P^{n_{0}}<P_{0}$, for some $\left.n_{0}\right\rangle 0$, where $P^{n_{0}}=\left\langle g^{n_{0}}\right.$ : $g \in P\rangle$. We deduce that $F_{0}$ is parabolic if and only if the rank of $P_{0}$ is equal to $t-1$, the rank of $P$. To show that $F^{\prime}$ is not an $m$-subgroup it is sufficient therefore to prove the existence of a nonzero $\mathbf{Z} G$-submodule of $P$ whose rank is strictly less than $t-1$, where, as before, $G=\Gamma / F^{*}$. Such a submodule exists when $F^{u}$ is not a maximal subgroup of $\Gamma[6$, p. 455].

More precisely, if $F^{u}$ is not maximal, the permutation group $\Gamma / F^{*}$, which acts on $p_{1}, \ldots, p_{t}$ as described above, is imprimitive. Let $\psi_{1}(\subseteq\{1, \ldots, t\})$ denote a block and let $\left\{\psi_{1}, \ldots, \psi_{s}\right\}$ be the corresponding complete block system. The elements $\Pi_{j \in \psi_{i}} p_{j}, 1 \leqslant i \leqslant s$, together with those of $F^{\prime}$, generate a nonparabolic normal subgroup of $\Gamma$ which properly contains $F^{\prime}$.

We assume from now on that $F^{u}\left(=\left(F^{*}\right)^{u}\right)$ is maximal. Since $F \neq \Gamma^{\prime}, F^{*}$ $\left(\neq \Gamma^{\prime}\right)$ is free and so by Lemma 2.1 we may assume also that $F=F^{*}$. By Lemma 2.2 and the above hypotheses we deduce that $F$ has level 6 and cusp number $q(\in S)$. In this case [8] we have $\Gamma^{\prime \prime}=\Delta(6)<F<\Gamma^{\prime}$, so that $P=\Gamma^{\prime \prime} / F^{\prime}$. Further $[8], \Gamma / F$, represented as a permutation group on the left cosets of $F^{u} / F$, is a Frobenius group (as is implied by [7, Theorem 4.2]). We now apply the results of $\$ 3$.

By Lemma 3.8, $F^{\prime}$ is not an $m$-subgroup when $q=p^{2}$ and so we now confine our attention to the case $q=p$. Using Newman's notation [8], $\Gamma^{\prime}=\langle a, b\rangle$ is a free group of rank 2 and $F / \Gamma^{\prime \prime}$ is a free abelian group of rank 2 generated by $b^{p}$ and $a b^{m}\left(\bmod \Gamma^{\prime \prime}\right)$, where $m^{2}+m+1 \equiv 0(\bmod p)$. Further $\Gamma^{\prime} / F$ is a cyclic group of order $p$ which is generated by $b(\bmod F)$. Since $t(F)=p$, it is clear that any parabolic element of $F$ is conjugate in $F$ to a power of one and only one of the following: $u^{6}, b u^{6} b^{-1}, \ldots, b^{p-1} u^{6} b^{-p+1}$. (Note that $t\left(\Gamma^{\prime}\right)=1$.) By considering the canonical presentation for $F$ given at the start of this section, it is clear that the only relation $\left(\bmod F^{\prime}\right)$ satisfied by these parabolic elements is

$$
\prod_{i=0}^{p-1} b^{i} u^{6} b^{-i}=1 \quad\left(\bmod F^{\prime}\right)
$$

Let $C / F^{\prime}$ be the centre of $\Gamma^{\prime} / F^{\prime}$ and suppose that $C \cap \Gamma^{\prime \prime} \neq F^{\prime}$. By Lemma 3.5, $C$ is parabolic and so there exists $r \neq 0$ such that $b u^{6 r} b^{-1} \equiv u^{6 r}$ $\left(\bmod F^{\prime}\right)$, which contradicts the above assertion. Hence $C \cap \Gamma^{\prime \prime}=F^{\prime}$ so that 
$C$ is nonparabolic. Further $C \neq F^{\prime}$ since $b^{p} \in C$, and so $F^{\prime}$ is not an m-subgroup.

After McCarthy [3] we say that an infinite group is just-infinite if all its proper quotients are finite. For example, $\Gamma / \Gamma(2)^{\prime}$ and $\Gamma / \Gamma(3)^{\prime}$ are just-infinite ([4], [11]).

TheOREM B. In the above notation, $C$ is an $m$-subgroup. Furthermore, $\Gamma / C$ is just-infinite.

Proof. Let $D\left(\neq F^{\prime}\right)$ be any nonparabolic normal subgroup of $\Gamma$ containing $F^{\prime}$ and let $L$ denote $D \Gamma^{\prime \prime}$. The level of $L$ divides 6 and $L^{\prime} \leqslant D$.

If $L$ is not free $\left(\Gamma: L^{\prime}\right)$ (and hence $(\Gamma: D)$ ) is finite [9, p. 143] which implies that $D$ is parabolic. If $L=\Gamma(2), D=\Gamma(2)^{\prime}$ [4] which implies that $\Gamma(2) \leqslant \Gamma^{\prime}$. Hence $L \neq \Gamma(2)$ and, similarly [11], $L \neq \Gamma(3)$. We conclude that the level of $D \Gamma^{\prime \prime}$ is 6 in which case [8] $D \leqslant \Gamma^{\prime}$.

Since $\left(\Gamma^{\prime}: F\right)=p$, we have $D F=\Gamma^{\prime}$ or $F$. However, $D F=\Gamma^{\prime}$ implies that $\Gamma^{\prime \prime}=\Delta(6) \leqslant D$. It follows that $D \leqslant F$. If $D \leqslant \Gamma^{\prime \prime}$ then $D \cap \Gamma^{\prime \prime}=D$. But by Lemma 3.5, $D \cap \Gamma^{\prime \prime}=F^{\prime}$, which contradicts the fact that $D \neq F^{\prime}$. We deduce [8] that $\left(\Gamma: D \Gamma^{\prime \prime}\right)$ is finite and that $D / F^{\prime}$ is free abelian of rank 2 .

$\Gamma / D_{\mathrm{Q}}$ is a subgroup of $\operatorname{Aut}\left(D / F^{\prime}\right)(\cong \mathrm{GL}(2, \mathbf{Z}))$, where $D_{0} / F^{\prime}=$ $C_{\Gamma / F^{\prime}}\left(D / F^{\prime}\right)$. Clearly $F \leqslant D_{0}$ and, since $\operatorname{GL}(2, \mathbf{Z})$ has no elements of order $p>3, \Gamma^{\prime} \leqslant D_{0}$. We conclude that $D \leqslant C$ which implies that $C$ is an $m$-subgroup.

To show that $\Gamma / C$ is just-infinite let $C_{0}$ be any normal subgroup of $\Gamma$ containing $C$. We note that $\left(\Gamma: C \Gamma^{\prime \prime}\right)$ is finite since $C \cap \Gamma^{\prime \prime}=F^{\prime}$ and $C \neq F^{\prime}[8]$. By Lemma 3.5, we have

$$
\text { (a) } C_{0} \cap \Gamma^{\prime \prime}=F^{\prime} \text { or } \quad\left(\text { b) }\left(\Gamma^{\prime \prime}: C_{0} \cap \Gamma^{\prime \prime}\right)<\infty\right. \text {. }
$$

(a) If $C_{0} \cap \Gamma^{\prime \prime}=F^{\prime}$ then $\left(C_{0}: C\right)=\left(C_{0}: C \cdot\left(C_{0} \cap \Gamma^{\prime \prime}\right)\right)=\left(C_{0} \Gamma^{\prime \prime}: C \Gamma^{\prime \prime}\right)$ is finite. Hence $C_{0}$ is nonparabolic and so by the first part of the proof of this theorem $C_{0}=C$.

(b) If $\left(\Gamma^{\prime \prime}: C_{0} \cap \Gamma^{\prime \prime}\right)=\left(C_{0} \Gamma^{\prime \prime}: C_{0}\right)$ is finite, then so is $\left(\Gamma: C_{0}\right)$.

3. Integral representations of Frobenius groups. Let $F$ be a normal subgroup of $\Gamma$ of level 6 and cusp number $q$, where $q$ is as defined above. Using [8] it can be shown that $F=F^{*}$ and that $\Gamma / F$, represented as a permutation group on the left cosets of $F^{u} / F$, is a Frobenius group with cyclic complement $F^{u} / F$ of order 6 and elementary abelian kernel $\Gamma^{\prime} / F$ of order $q$. We put $G=\Gamma / F, H=F^{u} / F$ and $K=\Gamma^{\prime} / F$.

LEMMA 3.1 ([2, p. 336]). Let $h$ be an element of $H$ of order 2. Then $h k h^{-1}=k^{-1}$ for all $k \in K$.

LEMMA 3.2 ([2, p. 143]). A complete set of inequivalent irreducible representations of $G$ (over $C$ ) consists of 6 representations of degree 1 and $(q-1) / 6$ of degree 6. The representations of degree 6 are faithful. 
We use the numbers $1, \ldots, q$ to label the left cosets of $H$ which are permuted by $G$ as described above. If $1 \leqslant i \leqslant q$ and $g \in G$, let $g(i)$ be the image of $i$ under the action of $g$. Let $V$ be the $\mathrm{Z} G$-module of rank $q$ with integral basis $\mathbf{v}_{1}, \ldots, \mathbf{v}_{q}$ and with the action of $G$ defined by $g \mathbf{v}_{i}=\mathbf{v}_{g(i)}$. Let $M=V / V_{0}$, where $V_{0}$ is the $\mathbf{Z} G$-submodule of $V$ generated by $\mathbf{v}_{1}$ $+\cdots+v_{q}$. It is clear that we have the following result.

LEMMA 3.3. $M$ is a ZG-module of rank $q-1$ which is isomorphic to $P=F^{\prime} \cdot \Delta(6) / F^{\prime}=\Gamma^{\prime \prime} / F^{\prime}$ (in the notation of $\S 2$ ).

LEMMA 3.4. Every nonzero ZG-submodule of $M$ is faithful and its rank is divisible by 6 .

Proof. We prove that $M$ has no $\mathrm{ZG}$-submodules of rank 1 . The result will then follow from Lemma 3.2.

Let $\left\langle\mathbf{v}, \mathbf{v}_{0}\right\rangle$ be a $Z G$-submodule of $V$, where $v_{0}=v_{1}+\cdots+v_{q}$. This gives rise to a 2-dimensional representation of $G$ by means of integral matrices which are of the form $\left[\begin{array}{ll}1 & 0 \\ 0 & 1\end{array}\right]$ or $\left[\begin{array}{cc}-1 & 0 \\ * & 1\end{array}\right]$. It follows that $g^{2} v=v$ for all $g \in G$. We deduce that $g \mathbf{v}=\mathbf{v}$ for all $g \in K$.

Since each nonidentity element of $K$ "fixes" no symbols, $\{k(1): k \in K\}=$ $\{1, \ldots, q\}$, i.e., $K$ acts transitively. We conclude that $\mathbf{v} \in\left\langle\mathbf{v}_{0}\right\rangle$.

We recall [1, p. 497] that a nonzero $\mathbf{Z} G$-module is said to be $\mathbf{Z}$-irreducible if it contains no nonzero $\mathbf{Z} G$-submodules of strictly smaller rank; otherwise it is said to be $\mathbf{Z}$-reducible.

LEMMA 3.5. If $q=p, p$ prime, $p \equiv 1(\bmod 6)$, then $P$ is Z-irreducible.

Proof. By Lemma 3.3 it is sufficient to prove that $M$ is irreducible. Let $M_{0}$ be a nonzero $\mathrm{Z} G$-submodule of $M$ of rank $r(\leqslant q-1)$. By Lemma 3.4, $M_{0}$ is faithful. Let $A$ be a nonidentity integral matrix of degree $r$ representing an element of order $p$ in $G$. The roots of the characteristic polynomial of $A$ are $p$ th roots of unity and are not all equal to 1 . Since $A$ is an integral matrix its characteristic polynomial is invariant under the action of the elements of the Galois group of $\mathbf{Q}(\theta)$ over $\mathbf{Q}$, where $\theta$ is a primitive $p$ th roots of unity. Hence all the primitive $p$ th roots of unity are roots of the characteristic polynomial and so $r \geqslant p-1$. We conclude that $r=q-1$ so that $M$ (and hence $P$ ) is Z-irreducible.

For the remaining lemmas we make the following assumption:

$$
q=p^{2}, \quad p \text { prime }, \quad p \equiv-1(\bmod 6) \text {. }
$$

We choose $x, y, z \in G$ such that $G=\langle x, y, z\rangle, K=\langle x, y\rangle$ and $H=\langle z\rangle$.

LEMMA 3.6. Assume (*). Let $G$ permute the numbers $1, \ldots, q$ as described above. With respect to this action $\langle x\rangle$ has $p$ orbits $X_{1}, \ldots, X_{p}$, say, each of size p.

Proof. Since $G$ is Frobenius with kernel $K, x$ does not "fix" any symbol. 
Lemma 3.7. Assume (*). Then $G$ permutes the elements of the set

$$
\mathfrak{X}=\left\{X_{i}, z\left(X_{i}\right), z^{2}\left(X_{i}\right): 1 \leqslant i<p\right\} .
$$

Proof. Since $x, y$ commute, $\langle y\rangle$ permutes the orbits of $\langle x\rangle$, i.e. the elements of the set $\left\{X_{i}: 1 \leqslant i \leqslant p\right\}$, and hence so does $K=\langle x, y\rangle$. Since $K \varangle G$ it follows that $K$ also permutes the elements of each of the sets $\left\{z\left(X_{i}\right)\right.$ : $1<i<p\}$ and $\left\{z^{2}\left(X_{i}\right): 1 \leqslant i \leqslant p\right\}$. Thus $K$ permutes the elements of $\mathfrak{X}$.

By Lemma 3.1, $z^{3} x z^{-3}=x^{-1}$ so that, for $1 \leqslant i \leqslant p, z^{3}\left(X_{i}\right)=X_{j}$, for some $j$. Thus $z$ permutes the elements of $\mathfrak{X}$ and hence so does $G=\langle K, z\rangle$.

Lemma 3.8. Assume (*). Then $P$ is $\mathbf{Z}$-reducible. More precisely, $P$ contains a nonzero $\mathrm{Z} G$-submodule of rank at most $3(p-1)$.

Proof. By Lemma 3.3 it is sufficient to prove that these assertions hold for $M$. Let $M_{1}$ be the $\mathbf{Z}$-submodule of $M$ which is generated by the images of the $3 p$ elements of $V, \mathbf{x}_{i}, z \mathbf{x}_{i}, z^{2} \mathbf{x}_{i}(1 \leqslant i \leqslant p)$, where $\mathbf{x}_{i}=\sum_{j \in X_{i}} \mathbf{v}_{j}$. Since $\sum_{i=1}^{p} \mathbf{x}_{i}$ $=\sum_{j=1}^{q} \mathbf{v}_{j} \in V_{0}, M_{1}$ has rank at most $3(p-1)$. We recall that, for any $g \in G$, $g \mathbf{v}_{j}=\mathbf{v}_{g(j)}$. It follows from Lemma 3.7 that $M_{1}$ is a $\mathbf{Z} G$-module, as required.

Although the final lemma is not required in the proof of Theorem $\mathbf{A}$ we include it for completeness.

LEMMA 3.9. In the above notation, $M_{1}$ is a $\mathrm{Z}$-irreducible $\mathrm{Z} G$-module of rank $3(p-1)$.

Proof. Let $M_{0}$ be a $\mathrm{Z}$-irreducible $\mathrm{Z} G$-submodule of $M$ of rank $r$, say. By Lemma 3.4, $M_{0}$ is faithful and 6 divides $r$.

We regard the representation of $G$ provided by $M_{0}$ as a Q-representation. This representation is irreducible over $Q$ in the usual sense $[1, p .497]$. By Clifford's Theorem [1, p. 343] (with base field Q), $M_{0}$ is a direct sum of conjugates of a $\mathbf{Q}$-irreducible $\mathbf{Q} K$-module $N$, say. Since $M_{0}$ is faithful, $N$ is nontrivial. Let $k$ be an element of order $p$ which acts nontrivially on $N$. By Clifford's Theorem, $N$ is a direct sum of conjugate nontrivial $\mathbf{Q}$-irreducible representations of $\langle k\rangle$. But by [1, p. 282, Ex. 2] such representations have degree $p-1$. Thus $p-1$ divides the rank of $N$ and hence $p-1$ divides $r$. Since $p \equiv-1(\bmod 6)$ and $6 \mid r$ it follows that $3(p-1)$ divides $r$. The result follows since by Lemma 3.8 the rank of $M_{1}$ is at most $3(p-1)$.

\section{REFERENCES}

1. C. W. Curtis and I. Reiner, Representation theory of finite groups and associative algebras, Pure and Appl. Math, vol. 11, Interscience, New York, 1962.

2. D. Gorenstein, Finite groups, Harper and Row, New York, 1968.

3. D. McCarthy, Infinite groups whose proper quotient groups are finite, I, Comm. Pure Appl. Math. 21 (1968), 545-562.

4. W. Magnus, Rational representations of Fuchsian groups and non-parabolic subgroups of the modular group, Nachr. Akad. Wiss. Göttingen Math.-Phys. K1. II 1973, 179-189.

5. A. W. Mason, Lattice subgroups of free congruence groups, Glasgow Math. J. 10 (1969), $106-115$. 
6. , Lattice subgroups of normal subgroups of genus zero of the modular group, Proc. London Math. Soc. (3) 24 (1972), 449-469.

7. A. W. Mason and W. W. Stothers, Remarks on a theorem of Leon Greenberg, Pacific J. Math. 78 (1978), 385-395.

8. M. Newman, A complete description of the normal subgroups of genus one of the modular group, Amer. J. Math 86 (1964), 17-24.

9. __ Integral matrices, Academic Press, New York and London, 1972.

10. P. E. Schupp, Embeddings into simple groups, J. London Math. Soc. (2) 13 (1976), 90-94.

11. C. Tretkoff, Non-parabolic subgroups of the modular group, Glasgow Math. J. 16 (1975), 91-102.

Department of Mathematics, Universtty of Glasgow, Glasgow G12 8QW, Scotland 